Check for updates

Cite this: RSC Adv., 2018, 8, 2377

Received 10th November 2017 Accepted 2nd January 2018

DOI: $10.1039 / c 7 r a 12273 j$

rsc.li/rsc-advances

\section{A computational study of energy barriers of structural transformations and hydrogen transfer in boehmite}

\begin{abstract}
Yingjian Jiang, (D) Yaoping Xie and Haibo Guo*
The crystal structure of boehmite $(\gamma-\mathrm{AlOOH})$ contains a large amount of hydrogen bonds that are joined into chains by sharing hydrogen-bond donor and acceptor oxygen atoms. The hydrogen ions in the hydrogen-bond chains are highly mobile and have complicated structural characterizations, and this feature may well be utilized for proton-conducting applications, but the mechanism is unknown without the dynamic parameters of the hydrogen-transfer processes. We propose probable hydrogen-transfer paths and compute their energy barriers using density functional theory with van der Waals density functionals, on both perfect and vacancy-containing crystal structures. It is revealed that the energy barriers are generally below $21 \mathrm{~kJ} \mathrm{~mol}^{-1}$ in a perfect crystal, and $14 \mathrm{~kJ} \mathrm{~mol}^{-1}$ in a vacancy-containing structure. The low energy barriers are indicators of the high proton conductivity of boehmite even at room temperature.
\end{abstract}

\section{Introduction}

Boehmite $(\gamma$-AlOOH) is an aluminum oxyhydroxide, metastable relative to diaspore $(\alpha-\mathrm{AlOOH})$ under ambient conditions. Boehmite occurs naturally as one of the main components of bauxite ore, ${ }^{1,2}$ and has been studied by various research groups for multiple applications, including catalysts, ${ }^{3}$ adsorbents, ${ }^{4-7}$ and thin porous membranes. ${ }^{8}$ For these applications, boehmite is frequently used as a precursor for manufacturing alumina phases, such as $\gamma-\mathrm{Al}_{2} \mathrm{O}_{3}$ and $\alpha-\mathrm{Al}_{2} \mathrm{O}_{3}$, usually through thermal dehydration. ${ }^{9}$ Less explored, however, is that boehmite itself may serve as a proton conductor for electrochemical reactions in aqueous environments.

Recently, a particularly large amount of work has been focused on proton-conducting materials ${ }^{10}$ which have many applications in the electrochemical industry. For instance, proton exchange membrane (PEM) fuel cells (PEMFC) rely critically on proton conductors, in form of thin membranes, for charge and mass transport. The general requirements of a good electrolyte membrane for PEMFCs are: high proton conductivity $\left(>10^{-2} \mathrm{~S} \mathrm{~cm}^{-1}\right)$, good chemical stability in highly acidic media and thermal stability at low to medium temperatures, low cost, and ability to be processed into thin films. ${ }^{11}$ Many PEMs have been developed, some being commercialized, but no membranes meet all the criteria. Commercially available products like Nafion developed by Dupont de Nemours also have some limitations, among which the most serious one is that proton conductivity drops significantly if operating

School of Materials Science and Engineering, Shanghai University, Shanghai, China. E-mail: guohaibo@shu.edu.cn temperature is above $90{ }^{\circ} \mathrm{C} \cdot{ }^{12,13}$ Therefore, finding alternative materials to improve or replace currently available PEMs is an important aim of numerous researches about PEMs.

Previous studies have revealed that hydrogen bonding plays an important role in proton conduction mechanisms. ${ }^{14,15}$ Thus, those structures that contain a large amount of hydrogen bonds are likely good proton conductors. Boehmite has a similar hydrogen-bond network as water, which was deemed to be an excellent proton conductor. ${ }^{16}$ It is of technological interest to investigate the proton-conducting ability of the hydrogen-bond network in boehmite. Since boehmite dehydrate at about $400{ }^{\circ} \mathrm{C}$ (variable with samples and moisture), ${ }^{17}$ much higher than that of boiling temperature of bulk water under ambient pressure, it is possible to prepare proton exchange membranes that can work at higher temperatures than current Nafion membranes. Elevated working temperatures in the context of PEMFCs mean improved reaction kinetics of the electrochemical reactions, and reduced chance of catalyst poisoning. Other advantages of boehmite include its low cost and well-developed synthesis methods. With all these advantages, the proton conductivity of boehmite, however, has seldom been measured, partly because boehmite is highly anisotropic and protons may only diffuse along a specific direction.

In boehmite's crystal structure, hydrogen-bonds connect to one another and form chains along crystallographic [001] direction of the orthorhombic cell. These parallel hydrogenbond chains bind interlocking double layers of edge-sharing $\mathrm{AlO}_{6}$ octahedra that are stacked along [010] direction. The locations of $\mathrm{Al}$ and $\mathrm{O}$ atoms in boehmite have been well measured by X-ray diffraction (XRD) and neutron diffraction experiments. ${ }^{18,19}$ The locations of $\mathrm{H}$ atoms in boehmite, 
however, have been elusive for a long time in spite of multiple structural characterizations based on Raman, ${ }^{20,21}$ infrared (IR) ${ }^{22}$ and nuclear magnetic resonance (NMR) spectroscopy. ${ }^{23}$ Because $\mathrm{H}$ atoms are weak X-ray scatters, it is difficult to locate the $\mathrm{H}$ atoms in boehmite from XRD experiments. The crystal structure of boehmite was deduced to be in the space group $\mathrm{Cmcm}$ from the definite positions of $\mathrm{Al}$ and $\mathrm{O}$ atoms. The neutron diffraction technique, which has much higher resolution in measuring $\mathrm{H}$ atoms' positions than XRD, confirms the structural characterization results by XRD. The previous researches on synthetic lepidocrocite $(\gamma \text {-FeOOH })^{24,25}$ also attributed the structure to the Cmom group.

In the unit cell of boehmite, two probable positions of hydrogen atoms are found to be compatible with the space group $\mathrm{Cmcm}$ in boehmite. The hydrogen atoms may reside in Wyckoff site $8 e$, forming $\mathrm{O}-\mathrm{H}$ bonds that are perpendicular to the double-sheet planes. This is highly unlikely because it doesn't conform to the oxygen's orthogonal bonding structure, and researchers have not found significant electron density at this position. ${ }^{26}$ Ewing $^{25}$ proposed that the hydrogen atom is located in Wyckoff site $4 a$, the midpoint tween two interlayer oxygen atoms. By this way, symmetric $\mathrm{O}-\mathrm{H}-\mathrm{O}$ hydrogen bonds are formed. This structure is, however, not acceptable because of the exceptionally long bond length of the symmetric $\mathrm{O}-\mathrm{H}-\mathrm{O}$ bonds, approximately $2.67 \AA$, which is apparently larger than $2.55 \AA$ for most of confirmed symmetric $\mathrm{O}-\mathrm{H}-\mathrm{O}$ bonds. ${ }^{27}$

Several space groups conforming to asymmetric $\mathrm{O}-\mathrm{H} \cdots \mathrm{O}$ hydrogen bonds have been considered, such as $C m c 2_{1},{ }^{28} P c a 2_{1}$ and $P 2_{1} / c{ }^{29}$ The only real difference among these structures is the relative positions of the $\mathrm{H}$ atoms. Most of previous studies focus on relative energetic stabilities of the probable crystal structures of boehmite. Some questions, like whether the above crystal structures can transform into each other and how defects affect the structural transformations, are unsolved. Hence, we address the structural transformations for both perfect crystal structure and crystal structures with defects. In the first part, we investigate the $C m c 2_{1}$ and $P c a 2_{1}$ space groups, as proposed by previous studies, and propose a new space group of $P m c 2_{1}$ for boehmite. For the second part, we focus on the defects of hydrogen vacancies for simplicity. The structural transformations involve breakage and formation of $\mathrm{O}-\mathrm{H}$ bonds in the hydrogen-bond chains, so we calculate energy barriers for these processes to assess the easiness (or difficulty) of the transformations.

These short-distance displacements of hydrogen atoms in boehmite, when combined with the long hydrogen-bond chains, may result in long-distance displacement of the hydrogen atoms, following the Grotthuss hopping mechanism. ${ }^{30}$ By calculating the energy barriers for the structural transformations, we are able to estimate proton conductivity as the two properties are tightly correlated. Proton exchange membranes are primarily characterized by proton conductivity. ${ }^{31}$ Before extensive experimental measurement of the proton conductivity, it is very useful of computational studies to assess probable mechanisms of hydrogen transfer in this highly anisotropic material.

\section{Methodology}

In this study we investigate thermodynamic stability of boehmite in space groups $C m c 2_{1}$ (space group no. 36), ${ }^{28} \mathrm{Pmc2}_{1}$ (space group no. 26), and $P c a 2_{1}$ (space group no. 29) ${ }^{29}$ by comparing their total energies. Energy barriers associated with proton-transfer paths are calculated using the climbing-image nudged elastic band (CI-NEB) method implemented in VTST code. $^{32,33}$ All the calculations are done using density functional theory (DFT) implemented in Vienna $A b$ initio Simulation Package (VASP). ${ }^{34-36}$ The electronic exchange and correlation interactions are described by the PBE (Perdew-Burke-Ernzerhof) functionals, ${ }^{37}$ and van der Waals density functionals (vdW$D F)^{38-40}$ combined with exchange part of PBE, referred to as vdW-PBE hereafter. The effects of nuclei and core electrons are described using the Projector Augmented Wave (PAW) method. ${ }^{\mathbf{4 1 , 4 2}}$ The PAW datasets for elements $\mathrm{Al}, \mathrm{O}$, and $\mathrm{H}$ are from the PAW dataset library shipped with VASP. The core radii are 1.90 Bohr for $\mathrm{Al}, 1.52 \mathrm{Bohr}$ for $\mathrm{O}$, and 1.10 Bohr for $\mathrm{H}$.

As for computational settings, the cut-off energy of plane wave functions is $500 \mathrm{eV}$, electronic state occupations are modeled using the Gaussian smearing method with a small smearing width of $0.005 \mathrm{eV}$, and total energies are extrapolated to zero electronic temperature. Sampling in the first Brillouin zone is done on special $k$-points generated according to the Monkhorst-Pack method ${ }^{43}$ using a regular grid of $8 \times 8 \times 6$ for the primitive cell of the $C m c 2_{1}$ structure, $10 \times 6 \times 8$ for the $P m c 2_{1}$ structure, and $6 \times 6 \times 8$ for the $P c a 2_{1}$ structure, according to tests by a convergence criterion of $0.001 \mathrm{eV}$ per formula unit. The numbers of irreducible $k$-points are 60, 60, and 36 for the $C m c 2_{1}, P m c 2_{1}$, and $P c a 2_{1}$ structures, respectively. The convergence criteria are set to $1.0 \times 10^{-7} \mathrm{eV}$ for energy differences in self-consistent-field iterations, and $0.005 \mathrm{eV}^{-1}$ for residual Feynman-Hellmann forces in relaxations.

Structures with hydrogen vacancies are generated by removing one hydrogen atom from $2 \times 1 \times 2$ and $3 \times 1 \times 2$ supercells with respect to the conventional cell of $C m c 2_{1}$. We use two supercells (instead of one) to check the interactions between vacancies of image cells in the periodic boundary setting. The grids for generating $k$-points in the MonkhorstPack scheme are $8 \times 4 \times 8$ for the $2 \times 1 \times 2$ supercell, and $4 \times 2$ $\times 8$ for the $3 \times 1 \times 2$ supercell, resulting in 64 and 16 irreducible $k$-points, respectively.

For the transition-state calculations, we create 4 images between the starting and end structures by linear interpolations using a utility program of the VTST scripts. Our tests with 8 and 16 images for the transition from $C m c 2_{1}$ to $P m c 2_{1}$ show that the calculated energy barriers are already converged within $0.005 \mathrm{eV}$ for the calculations with 4 images. The force-based quick-min algorithm is used for finding minimum-energy path. Cell parameters are kept constant for all the transition states.

\section{Results and discussion}

\subsection{Crystal structures without defects}

We can clearly see that boehmite has a layered structure (see Fig. 1) that contains $\mathrm{AlO}_{6}$ octahedron layers and hydrogen-bond 
(a)

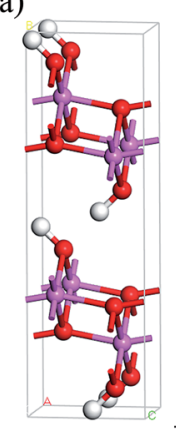

$\mathrm{Cmc}_{1}$ (b)

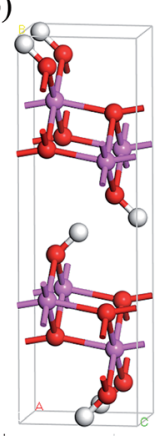

Pmc2 ${ }_{1}$ (c)

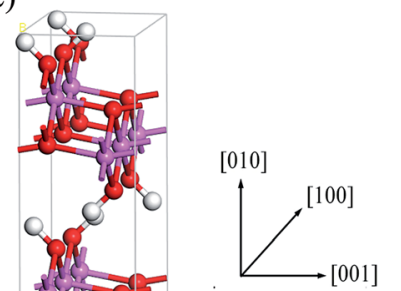

$\mathrm{Pca}_{1}$
Fig. 1 Crystal structures of boehmite oriented along the [100] direction. White atoms are $\mathrm{H}$, red atoms are $\mathrm{O}$, and pink atoms are Al. The structure in (a) is of $C m c 2_{1}$ symmetry, (b) is of $P m c 2_{1}$, and (c) is of $P c a 2_{1}$ space groups.

layers. Each hydrogen-bond layer is composed of parallel hydrogen-bond chains along the [001] crystallographic direction. The boehmite crystal structures of $C m c 2_{1}, P m c 2_{1}$ and $P c a 2_{1}$ space groups are all orthorhombic, and the main difference is orientations of the hydroxyl groups. Since around each oxygen atom in the hydrogen-bonding layers there are two positions at which a hydrogen atom may reside, corresponding to two possible configurations for a hydrogen-bond chain. For the $\mathrm{Cmc2}_{1}$ structure, all the hydroxyl groups $(\mathrm{O}-\mathrm{H})$ tilt towards the [001] direction; for the $P m c 2_{1}$ structure, the hydroxyl groups switch directions in adjacent hydrogen-bond layers; and for the $P c a 2_{1}$ structure, the hydroxyl groups in adjacent hydrogen-bond chains in a same layer have opposite directions.

The calculated lattice parameters of the $C m c 2_{1}$ structure are listed in Table 1, together with experimental values. By comparing the lattice parameters from plain PBE and vdW-PBE calculations, we find that the values for $a$ are almost the same (less than $0.01 \AA$ or $0.3 \%$ in differences) in both settings, the values for $c$ are also almost identical, and both are larger than the experimental values by about $1.1 \%$. As for the lattice parameter $b$ that is pertinent to interlayer distances, the value calculated by vdW-PBE is slightly smaller than the experimental value by about $0.01 \AA$, while the value by PBE differ from experimental value by about $0.1 \AA$ A In general, vdW-PBE performs better in reproducing the lattice parameters of boehmite.

It is noted that the orthorhombic cell of $\mathrm{Pca}_{1}$ is twice that of $C m c 2_{1}$ and $P m c 2_{1}$. The total energies of the three structures, i.e., $C m c 2_{1}, P m c 2_{1}$, and $P c a 2_{1}$, are compared to sort their relative thermodynamic stability. From Table 2 we can see that the

Table 1 Cell parameters of the $C m c 2_{1}$ structure. Each orthogonal cell contains 4 formula units $(Z=4)$. Unit: $\AA$

\begin{tabular}{llll}
\hline Reference & $a$ & $b$ & $c$ \\
\hline This work; PBE & 2.8946 & 12.1465 & 3.7361 \\
This work; vdW-PBE & 2.9021 & 12.2224 & 3.7325 \\
Experimental $^{44}$ & 2.8681 & 12.2336 & 3.6923
\end{tabular}

Table 2 Total energies of the $P m c 2_{1}$ and $P c a 2_{1}$ structures with respect to the $\mathrm{Cmc}_{1}$ structure. Unit: $\mathrm{kJ} \mathrm{mol}{ }^{-1}$

\begin{tabular}{lcc}
\hline Method & $P m c 2_{1}$ & $P c a 2_{1}$ \\
\hline PBE & $<0.01$ & -0.27 \\
vdW-PBE & $<0.01$ & -0.26 \\
\hline
\end{tabular}

$P m c 2_{1}$ structure has almost identical total energy as $C m c 2_{1}$ in both PBE and vdW-PBE calculations, but $P c a 2_{1}$ has consistently lower total energy than $C m c 2_{1}$ and $P m c 2_{1}$, by $-0.27 \mathrm{~kJ} \mathrm{~mol}^{-1}$ in PBE or $-0.26 \mathrm{~kJ} \mathrm{~mol}^{-1}$ in vdW-PBE calculations. Such small energy differences are close to resolution limits of experimental measurements and computations. The difference should be treated carefully because even though it is not negligible, the value is one order of magnitude smaller than the thermal fluctuation energy at room temperature (about $2.5 \mathrm{~kJ} \mathrm{~mol}^{-1}$ ). Thus, we show that the thermodynamic stability of $C m c 2_{1}$ and $P m c 2_{1}$ are essentially the same, and $P c a 2_{1}$ is slightly more stable than $C m c 2_{1}$ and $P m c 2_{1}$.

The order of thermodynamic stability of the three structures is related to the orientation of hydroxyl groups in hydrogenbond layers. In $C m c 2_{1}$ and $P m c 2_{1}$, all hydroxyl groups in

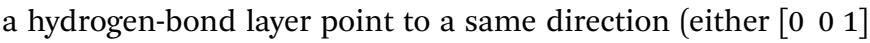
or $\left.\left[\begin{array}{lll}0 & 0 & 1\end{array}\right]\right)$, and have the same polarity. In $P c a 2_{1}$, hydroxyl groups in a hydrogen-bond layer point to opposite directions in alternating chains, and collectively have zero polarity. Interactions between electric dipoles of a same polarity increases the system's energy, while interactions between electric dipoles of opposite polarities are associated with a negative energy. It appears that dipolar interactions are negligible across neighboring hydrogen-bond layers, since the energies of $C m c 2_{1}$ and $P m c 2_{1}$ are almost identical. The interactions of dipole moments in adjacent chains in a same layer should be very weak, at the order of $0.1 \mathrm{~kJ} \mathrm{~mol}^{-1}$ that is readily overridden by thermal fluctuations at room temperature. We conclude that the three structures have the same thermodynamic stability within an error of $0.5 \mathrm{~kJ} \mathrm{~mol}{ }^{-1}$, and tentatively propose that hydroxyl polarity contributes slightly to their relative thermodynamic stability. In a same layer, the orientation of the hydroxyl groups in different zigzag-shaped chains should be independent of each other at normal conditions. This conclusion is consistent with a previous report in the literature. ${ }^{26}$

Because the three structures have almost the same thermodynamic stability, a boehmite sample may have domains of the three probable structures. The next question is whether they can transform into one another. If the structural transformations are easy at certain conditions, then the domains should be dynamic or volatile, otherwise the domains are static. We can assess energy barriers associated with the structural transformations, and compare the energy barriers with possible driving forces. Since the dipolar interactions between hydrogenbond chains are weak, it is convenient for us to assume that only one hydrogen-bond chain changes and all other chains are constant. We study the transformation between the $C m c 2_{1}$ and $P m c 2_{1}$ structures, and propose two paths for hydrogen atoms to cause this structural transformation: the stretch mode and the 
swing mode (see Fig. 2). In the stretch mode, all the hydroxyl bonds break and hydrogen atoms move to nearby oxygen atoms to form new hydroxyl bonds (Fig. 2a). In the swing mode, a hydrogen atom remains around a same oxygen atom, and the hydroxyl bond does not break but only changes its direction (Fig. 2b). Each mode requires cooperative motion of all hydrogen atoms in a whole hydrogen-bond chain, otherwise a high-energy state may occur that either has two hydrogen atoms bonded to one oxygen atom, or has a very small distance between two hydrogen atoms boned to two neighboring oxygen atoms. We point out that both modes can independently transform the structure between $C m c 2_{1}$ and $P m c 2_{1}$, but longrange transfer of hydrogen atoms is only possible with both modes cooperating in concert.

We use the CI-NEB method to calculate the energy barrier for the two hydrogen-transfer modes. As shown in Fig. 3, the PBE results indicate that the stretch mode has lower energy barrier $\left(13.52 \mathrm{~kJ} \mathrm{~mol}^{-1}\right)$ than the swing mode $\left(20.68 \mathrm{~kJ} \mathrm{~mol}^{-1}\right)$, while the vdW-PBE results show that the stretch mode and swing mode have almost the same energy barrier (18.70 and $18.26 \mathrm{~kJ} \mathrm{~mol}^{-1}$, respectively). The energy-barrier difference between these two modes is $7.16 \mathrm{~kJ} \mathrm{~mol}^{-1}$ for $\mathrm{PBE}$ and $0.44 \mathrm{~kJ} \mathrm{~mol}^{-1}$ for vdW-PBE, showing the influence the van der Waals forces on the transition states that involve hydrogen bonds in boehmite. Especially for the stretch mode, the energy barrier estimated by vdW-PBE is $5.18 \mathrm{~kJ} \mathrm{~mol}^{-1}$ higher than that by PBE. The large difference in energy barriers by the two methods can be understood by the arrangement of the atoms along the transition paths. In the stretch mode, the hydrogen atoms that are attached to one $\mathrm{AlO}_{6}$-octahedron layer move to another $\mathrm{AlO}_{6}$ layer, and this geometric change for the intermediate states is accompanied by energy increase by van der Waals interactions. In the swing mode, the hydrogen atoms are invariantly attached to one $\mathrm{AlO}_{6}$-octahedron layer along the whole transition path, for which the geometry change is smaller than that in the stretch mode.

Since the energy barriers are around $20 \mathrm{~kJ} \mathrm{~mol}^{-1}$, the energy required for transformation between the crystal structures can

(a)

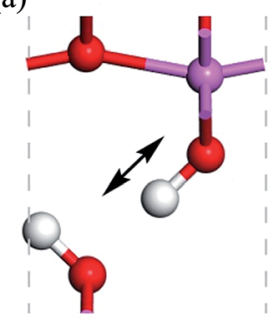

(b)

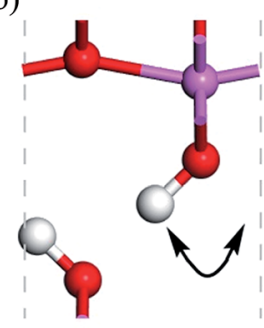

\section{Stretch}

\section{Swing}

Fig. 2 Proton-transfer modes in boehmite, with the direction of motions indicated by the arrows for the (a) stretch mode, (b) swing mode.

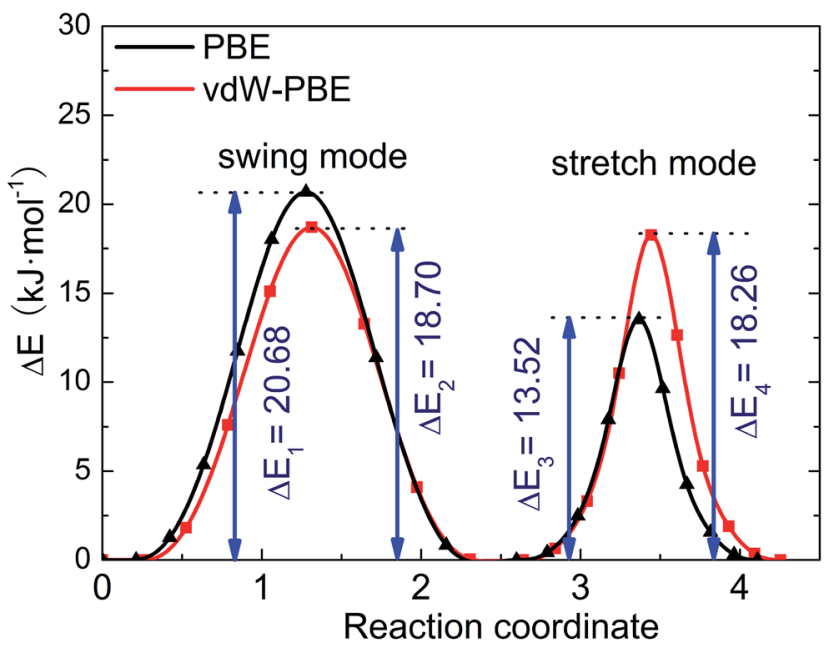

Fig. 3 Energy barrier of proton-transfer processes in boehmite. The reaction of coordinates are scaled for comparison.

be provided by thermal fluctuations. Energy fluctuations at temperature $T$ is roughly estimated to be $R T$ (where $R$ is gas constant, 8.31 $\mathrm{J} \mathrm{K}^{-1} \mathrm{~mol}^{-1}$ ). Even though it is thermodynamically metastable relative to diaspore $(\alpha-\mathrm{AlOOH})$ under ambient conditions, boehmite can be stable at temperatures below its dehydration temperature, about 700-750 K, before transforming into $\gamma-\mathrm{Al}_{2} \mathrm{O}_{3} \cdot{ }^{45-47}$ At $T=300 \mathrm{~K}, R T \approx 2.5 \mathrm{~kJ} \mathrm{~mol}^{-1}$, and at the dehydration temperature (around $750 \mathrm{~K}$ ), $R T \approx$ $6.2 \mathrm{~kJ} \mathrm{~mol}^{-1}$. In this temperature range (300-750 K), the energy barrier of the transition between $C m c 2_{1}$ and $P m c 2_{1}$ space groups is 3-8 times the $R T$ value. It turns out that this structural transition is highly probable even at room temperature.

We can use the Arrhenius equation to calculate structural transition rate as $^{48}$

$$
\nu=A \nu_{0} \exp \left(\frac{-\Delta E}{R T}\right),
$$

where $\nu$ stands for the frequency of successful structural transformation, $A$ is a prefactor that is assumed to be a systemdependent constant, $\Delta E$ is the energy barrier for the transition, $R$ is the gas constant, $T$ is the temperature, and $\nu_{0}$ is the attempt rate, which corresponds to the frequency of a characteristic phonon mode. The proton-transfer modes involve coordinative motion of atoms, and certain phonon vibration modes may assist the processes if the motion of atoms in the phonon modes coincides with that in the proton-transfer modes. Tunega et al. have obtained boehmite crystal's phonon spectra, ${ }^{48}$ where two $\mathrm{OH}$ vibration modes match the stretch and swing modes of proton transfer in the present study. The value of $\nu_{0}$ for the stretch mode $\left(2947 \mathrm{~cm}^{-1}\right)$ is about three times that of the swing mode $\left(1283 \mathrm{~cm}^{-1}\right)$. Assuming that the prefactor $A$ is the same for the two-transition modes, we can estimate the relative rate of the two transition modes. At room temperature (approximately 300 $\mathrm{K}$ ), for the energy barriers calculated using PBE,

$$
\frac{\nu_{\text {stretch }}}{\nu_{\text {swing }}} \approx 52.6
$$


and for the vdW-PBE case:

$$
\frac{\nu_{\text {stretch }}}{\nu_{\text {swing }}} \approx 3.6
$$

In both PBE and vdW-PBE calculations, the rate of stretchmode transitions is higher than swing-mode transitions. But, the rate differences and the causes are quite different in PBE and vdW-PBE calculations. In PBE, the rate of stretch-mode transitions is several tens of times of swing-mode transitions, and the difference is mainly caused by its smaller energy barrier; while in vdW-PBE, the rate of stretch-mode transitions is only about four times that of swing-mode transitions, and the difference is mainly caused by the characteristic phonon frequency.

\subsection{Crystal structure with defects of hydrogen vacancy}

Real boehmite crystals always contain various forms of defects, among which we concentrate on hydrogen vacancies. The normally continuous hydrogen-bond chains in a perfect lattice break at hydrogen vacancies, splitting into shorter chains. The hydrogen vacancies are expected to interrupt transition of nearby hydrogen atoms. In this study, we create hydrogen vacancies by removing a hydrogen atom from a supercell of boehmite, then design paths for hydrogen transfer to or from this vacancy position, and calculate energy barriers associated with these transition paths. Along the transition paths there are intermediate states for which the hydrogen atom in motion resides at one of the two equilibrium positions around an oxygen atom. In Fig. 4 we present five intermediate states, namely $\mathrm{VacH}$, VacH_s1, VacH_s1t2, VacH_t1 and VacH_t1s2. Two paths can be composed by connecting subsets of these states for transferring a hydrogen atom along the [001] or [001] direction. The first path is from VacH to VacH_s1 via a swing-mode motion of a nearby hydrogen atom, and to VacH_s1t2 via another stretch-mode motion of the hydrogen atom. By repeating this path, the hydrogen-vacancy moves continuously towards the [001] direction and, equivalently, the hydrogen atoms in this hydrogenbond chain move toward the [001] direction. The second path is from VacH to VacH_t1 via a stretch-mode motion of a nearby hydrogen atom, and to VacH_t1s2 via another swing-mode motion. The motion of the hydrogen vacancy and hydrogen atoms are both opposite to those in the first path.

These four steps involved in the two paths have different energy-barriers. We have used the CI-NEB method to calculate these energy-barriers and present the results in Table 3 and Fig. 5 . We can clearly see that the two paths have very similar highest energy barriers in both PBE and vdW-PBE calculations. From our PBE calculations, the swing-mode steps, i.e. from $\mathrm{VacH}$ to VacH_s1 and from VacH_t1 to VacH_t1s2, have higher energy barriers than the stretch-mode steps, i.e. from $\mathrm{VacH}$ to $\mathrm{VacH}+\mathrm{t} 1$ and from VacH_s1 to VacH_s1t2. The differences in these energy barriers are 1-2 $\mathrm{kJ} \mathrm{mol}^{-1}$. For vdW-BPE calculations, the stretch-mode steps have higher energy-barriers than the swingmode steps by approximately $6-8 \mathrm{~kJ} \mathrm{~mol}^{-1}$. The reasoning on the effects of vdW interactions on the stretch- and swing-modes has been discussed in the previous section for the perfect crystal, and also applies for the structures with hydrogen vacancies. The effects of hydrogen vacancies on the energybarriers will be discussed in the next paragraph.

By comparing the energy-barriers in Fig. 3 and Table 3, one finds that the hydrogen vacancy lowers energy-barriers of both

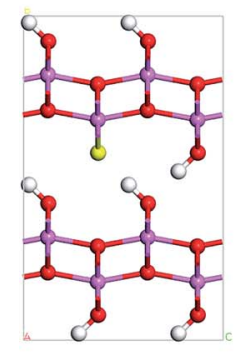

$\mathrm{VacH}$

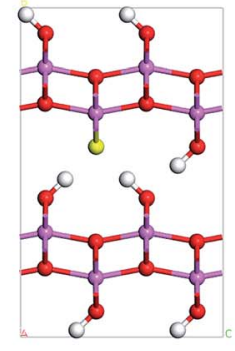

VacH_s1

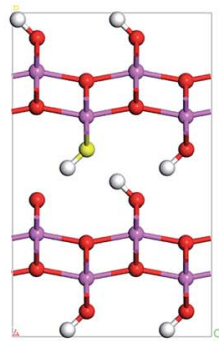

VacH_s1t2

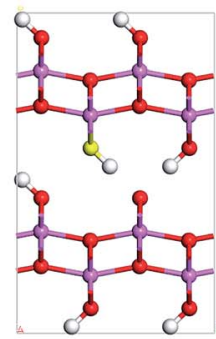

VacH_t1

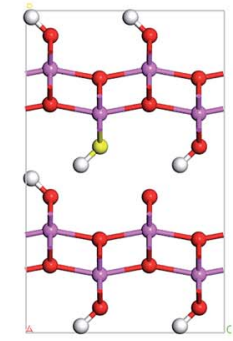

VacH t1s2

Fig. 4 Five states along proton-transfer paths in boehmite containing a hydrogen vacancy: VacH, VacH_s1, VacH_s1t2, VacH_t1 and VacH_t1s2. The yellow atom marks the position of the oxygen atom to which the missing hydrogen atom is bonded.

Table 3 The energy barriers of proton transfer processes. Unit: $\mathrm{kJ} \mathrm{mol}^{-1}$

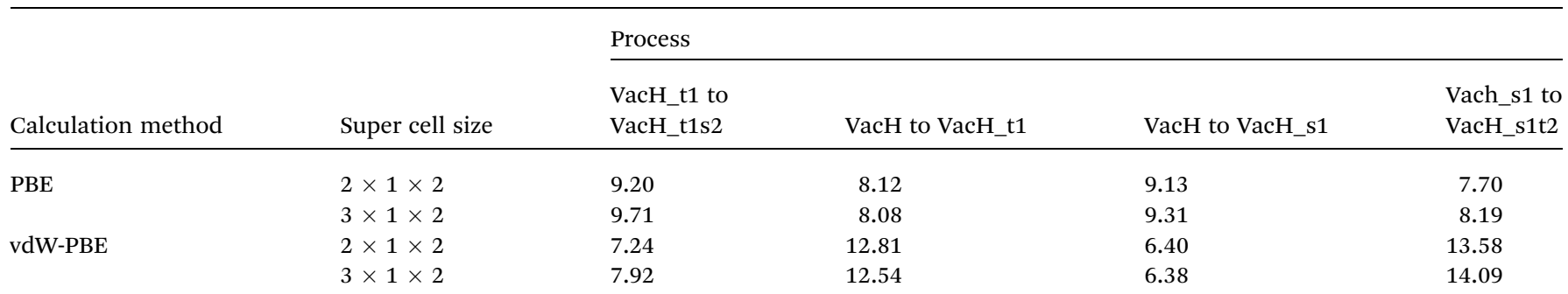




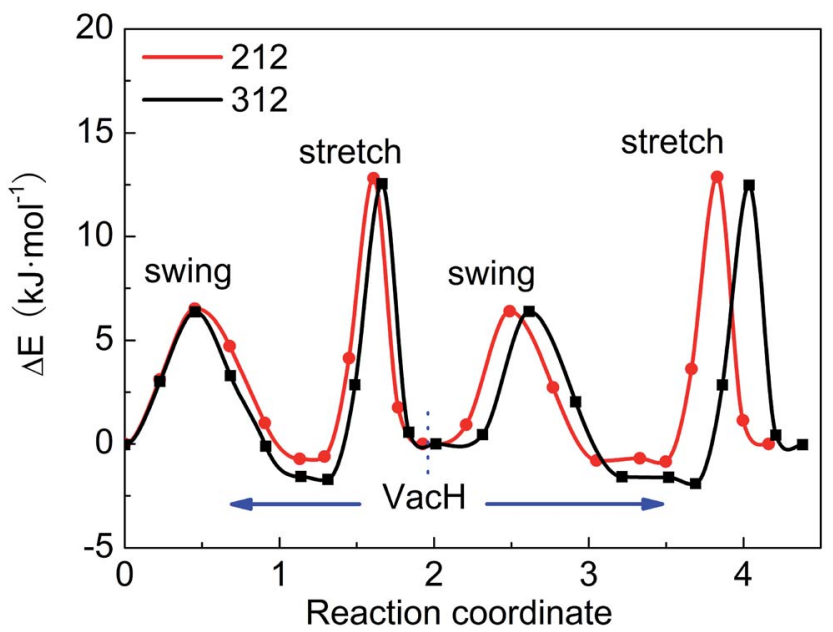

Fig. 5 Energy barriers for hydrogen-transfer path from state $\mathrm{VacH}+\mathrm{t} 1 \mathrm{~s} 2$ to $\mathrm{VacH} H \mathrm{t} 1, \mathrm{VacH}, \mathrm{VacH}$ _s1, then to $\mathrm{VacH}$ _s1t2, in supercell $2 \times 1 \times 2$ and $3 \times 1 \times 2$ using the vdW-PBE method stretch- and swing-modes, but to different extents. The energybarrier of the stretch mode is lowered by $4-6 \mathrm{~kJ} \mathrm{~mol}^{-1}$, and that of the swing mode is lowered by $10-12 \mathrm{~kJ} \mathrm{~mol}^{-1}$, both consistently in PBE and vdW-PBE calculations. The lowering in the energy barriers can be explained as follows. In a perfect crystal, all hydrogen atoms in a hydrogen-bond chain can only move coordinately to avoid the otherwise high-energy states. In the proximity of a hydrogen-vacancy, a hydrogen atom can move independently of other hydrogen atoms in either stretch or swing mode, requiring less energy than the motion of many hydrogen atoms in a perfect crystal. As for the difference in the vacancy-induced decreases of energy-barriers between the two modes (stretch and swing), it can tentatively be explained from the change in electrostatic interactions between the hydrogen ions. We assume the hydrogen ions carry the same amount of positive charge in a perfect crystal and vacancy-containing structures, thus the electrostatic interactions depend solely on distances between the ions. For simplicity, we only consider the electrostatic interaction between a hydrogen ion and its nearest

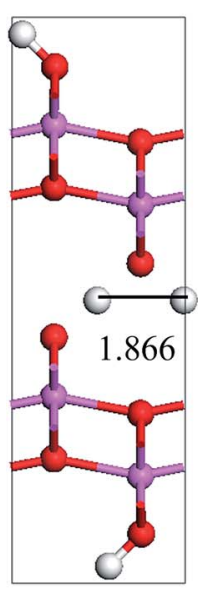

(a)

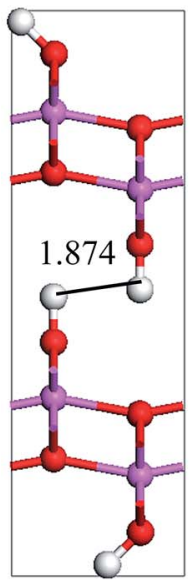

(c)

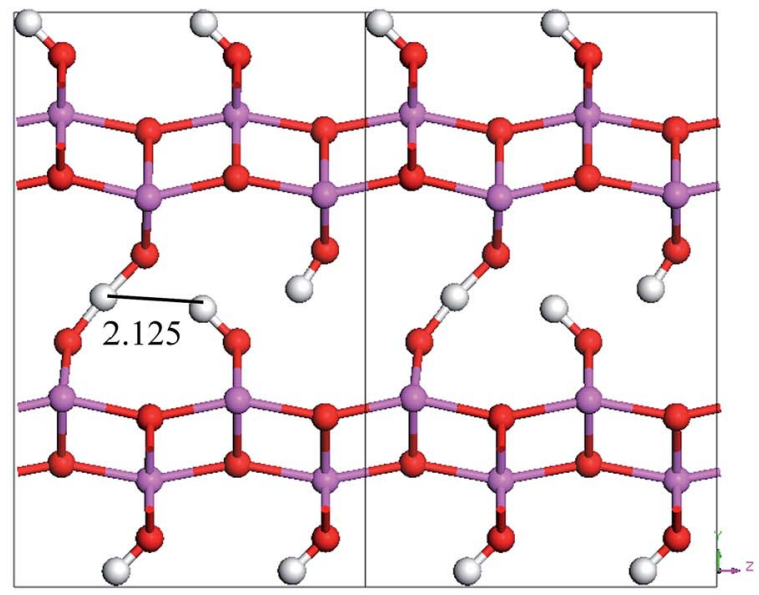

(b)

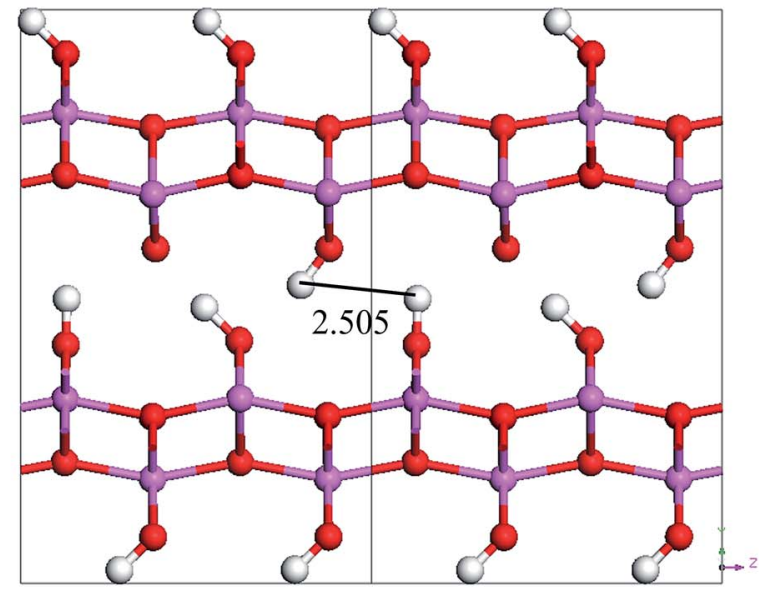

(d)

Fig. 6 Saddle-point structures in the transition paths and lengths tween neighboring hydrogen atoms in these structures. (a) is for the stretch-mode transition from $\mathrm{Cmc2}_{1}$ to $\mathrm{Pmc2}_{1}$, (b) is for the stretch-mode transition from VacH_s1 to VacH_s1t2, (c) is for the swing-mode transition from Cmc2 1 to $P m c 2_{1}$, and (d) is for the swing-mode transition from VacH to VacH_s1. The sizes of the supercells in (c) and (d) are $2 \times 1 \times 2$. The numbers are in unit of $\AA$. 
neighbor. Along each transition path the state of the highest energy, or the saddle point, can be identified, and some representative ones are shown in Fig. 6. For the saddle-point structures in the two stretch-mode transition paths, the distance between the nearest neighboring hydrogen ions changes from $1.866 \AA$ in a perfect crystal (Fig. 6a) to $2.125 \AA$ in the vacancycontaining structure (Fig. 6b). For the two swing-mode transition paths, the distance between the nearest neighboring hydrogen ions changes from $1.874 \AA$ in a perfect crystal (Fig. 6c) to $2.505 \AA$ in the vacancy-containing structure (Fig. 6d). Starting from similar distances between nearest neighboring hydrogen ions, the larger increase in the $\mathrm{H}-\mathrm{H}$ distances in the swingmode steps leads to larger decrease in the associated energy barriers.

We have tested two sizes of supercells, namely $2 \times 1 \times 2$ and $3 \times 1 \times 2$, in calculating the energy barriers in the vacancycontaining structures that, similar to many other defectcontaining structures, are expected to be sensitive to the supercells' sizes in the periodic boundary setting. For both PBE and vdW-PBE calculations, the differences between the energybarriers in the two supercells are below $1 \mathrm{~kJ} \mathrm{~mol}^{-1}$, as can be derived from the numbers in Table 3. The trends of the energy barriers with respect to the steps are also consistent (see Fig. 6). We are confident that the calculated energy barriers are converged with respect to the supercell's size at $2 \times 1 \times 2$.

\section{Conclusions and remarks}

In this paper, our computational results are mainly about thermodynamic stability of three probable structures of boehmite, and energy barriers for hydrogen transfer in boehmite's lattice with and without hydrogen vacancies. The results show that the $P c a 2_{1}$ structure has slightly lower total energy than the $C m c 2_{1}$ and $P m c 2_{1}$ structures due to different polarities of the $\mathrm{OH}$ bonds. Transfer paths of hydrogen atoms along the hydrogen-bond chains can be divided into steps of swing- and stretch-mode transitions, and are associated with different energy barriers that depend on computational settings. For a perfect crystal the energy barriers are below approximately $21 \mathrm{~kJ} \mathrm{~mol}^{-1}$, and for the structures with hydrogen vacancies, the energy barriers are below approximately $14 \mathrm{~kJ} \mathrm{~mol}^{-1}$. The computational settings of PBE and vdW-PBE lead to different orders of the energy barriers for swing-mode transitions and for stretch-mode transitions, in both perfect crystals and vacancy-containing structures. It has been observed van der Waals forces are important for hydrogen-bonds, ${ }^{49}$ thus the results of vdW-PBE should be more reliable and closer to reality.

The energy barriers are generally low in comparison with thermal fluctuation energies at room temperature and above, suggesting high mobility of hydrogen atoms in boehmite's lattice. Thus, the crystal structure of boehmite may comprise of all the three structures that are frequently changing into one another. It is difficult to identify domains of different polarity of the $\mathrm{OH}$ bonds without lowering the temperature. The average structure belongs to the $\mathrm{Cmcm}$ space group that is repeatedly confirmed by multiple experiments.
We suggest, based on the low energy barriers, that boehmite has a high proton conductivity that may be utilized for protonconducting applications. The proton transfer mechanism in boehmite do not rely on liquid-phase water, and the working temperature can be elevated to above $100{ }^{\circ} \mathrm{C}$. It has been stated in ref. 50 and 51 that a working temperature of $120^{\circ} \mathrm{C}$ and $50 \%$ or lower relative humidity are targets for the development for automotive use of hydrogen/air fuel cells. The only drawback is that the proton conductivity of boehmite is highly anisotropic. Synthesizing and assembling highly ordered boehmite crystals are challenging, but it should be much easier to make boehmite filler materials for organic membranes to enhance their hightemperature proton conductivity.

\section{Conflicts of interest}

There are no conflicts to declare.

\section{Acknowledgements}

This work is supported by Doctoral Fund of Ministry of Education of China (20133108120021), National Natural Science Foundation of China for Youths (51302166), and Municipal Natural Science foundation of Shanghai. The computations are performed on Compmat cluster and Ziqiang4000 supercomputer of the High Performance Center of Shanghai University.

\section{References}

1 J. T. Kloprogge, H. D. Ruan and R. L. Frost, J. Mater. Sci., 2002, 37, 1121-1129.

2 J. T. Kloprogge, L. V. Duong, B. J. Wood and R. L. Frost, J. Colloid Interface Sci., 2006, 296, 572-576.

3 K. V. P. M. Shafi, A. Ulman, J. Lai, N.-L. Yang and M.-H. Cui, J. Am. Chem. Soc., 2003, 125, 4010-4011.

4 R. Orlando, F. J. Torres, F. Pascale, P. Ugliengo, C. ZicovichWilson and R. Dovesi, J. Phys. Chem. B, 2006, 110, 692-701.

5 F. Granados-Correa, N. G. Corral-Capulin, M. T. Olguin and C. E. Acosta-Leon, Chem. Eng. J., 2011, 171, 1027-1034.

6 F. Granados-Correa and J. Jimenez-Becerril, J. Hazard. Mater., 2009, 162, 1178-1184.

7 B. Kasprzyk-Hordern, Adv. Colloid Interface Sci., 2004, 110, 19-48.

8 R. W. Hicks and T. J. Pinnavaia, Chem. Mater., 2003, 15, 7882.

9 R. W. N. Denigres, G. D. Rocha, C. R. Montes and A. C. VieiraCoelho, Mater. Res., 2016, 19, 659-668.

10 X. Meng, H. N. Wang, S. Y. Song and H. J. Zhang, Chem. Soc. Rev., 2017, 46, 464-480.

11 P. Ramaswamy, N. E. Wong and G. K. H. Shimizu, Chem. Soc. Rev., 2014, 43, 5913-5932.

12 S. S. Ivanchev, Russ. J. Appl. Chem., 2008, 81, 569-584.

13 S. Bose, T. Kuila, X. L. N. Thi, N. H. Kim, K. T. Lau and J. H. Lee, Prog. Polym. Sci., 2011, 36, 813-843.

14 K. D. Kreuer, S. J. Paddison, E. Spohr and M. Schuster, Chem. Rev., 2004, 104, 4637-4678.

15 T. Steiner, Angew. Chem., 2002, 114, 50-80. 
16 U. W. Schmitt and G. A. Voth, J. Chem. Phys., 1999, 111, 93619381.

17 S. J. Wilson, J. Solid State Chem., 1979, 30, 247-255.

18 A. N. Christensen, M. S. Lehmann and P. Convert, Acta Chem. Scand., Ser. A, 1982, 36, 303-308.

19 P. P. Reichertz and W. J. Yost, J. Chem. Phys., 1946, 14, 495501.

20 A. B. Kiss, G. Keresztury and L. Farkas, Spectrochim. Acta, Part A, 1980, 36, 653-658.

21 V. C. Farmer, Spectrochim. Acta, Part A, 1980, 36, 585-586.

22 M. C. Stegmann, D. Vivien and C. Mazieres, Spectrochim. Acta, Part A, 1973, 29, 1653-1663.

23 R. C. T. Slade and T. K. Halstead, J. Solid State Chem., 1980, 32, 119-122.

24 G. G. Christoph, C. E. Corbato, D. A. Hormann and R. T. Tettenhorst, Clays Clay Miner., 1979, 27, 81-86.

25 F. J. Ewing, J. Chem. Phys., 1935, 3, 420-424.

26 R. Hill, Clays Clay Miner., 1981, 29, 435-445.

27 A. Oles, A. Szytula and A. Wanic, Phys. Status Solidi, 1970, 41, 173-177.

28 J. D. Russell, V. C. Farmer and D. G. Lewis, Spectrochim. Acta, Part A, 1978, 34, 1151-1153.

29 Y. Noel, R. Demichelis, F. Pascale, P. Ugliengo, R. Orlando and R. Dovesi, Phys. Chem. Miner., 2009, 36, 47-59.

30 Q. Li, D. Aili, R. F. Savinell and J. O. Jensen, in High Temperature Polymer Electrolyte Membrane Fuel Cells: Approaches, Status, and Perspectives, ed. Q. Li, D. Aili, H. A. Hjuler and J. O. Jensen, Springer International Publishing, Cham, 2016, pp. 37-57, DOI: 10.1007/978-3-319-17082-4_3.

31 M. B. Herath, S. E. Creager, A. Kitaygorodskiy and D. D. DesMarteau, ChemPhysChem, 2010, 11, 2871-2878.

32 G. Henkelman, B. P. Uberuaga and H. Jonsson, J. Chem. Phys., 2000, 113, 9901-9904.

33 D. Sheppard, R. Terrell and G. Henkelman, J. Chem. Phys., 2008, 128, 134106.

34 G. Kresse and J. Furthmuller, Phys. Rev. B: Condens. Matter Mater. Phys., 1996, 54, 11169-11186.
35 G. Kresse and J. Furthmuller, Comput. Mater. Sci., 1996, 6, 15-50.

36 G. Kresse and J. Hafner, Phys. Rev. B: Condens. Matter Mater. Phys., 1994, 49, 14251-14269.

37 J. P. Perdew, K. Burke and M. Ernzerhof, Phys. Rev. Lett., 1996, 77, 3865-3868.

38 J. Klimes, D. R. Bowler and A. Michaelides, Phys. Rev. B: Condens. Matter Mater. Phys., 2011, 83, 13.

39 M. Dion, H. Rydberg, E. Schroder, D. C. Langreth and B. I. Lundqvist, Phys. Rev. Lett., 2004, 92, 4.

40 G. Roman-Perez and J. M. Soler, Phys. Rev. Lett., 2009, 103, 4.

41 P. E. Blöchl, Phys. Rev. B: Condens. Matter Mater. Phys., 1994, 50, 17953-17979.

42 G. Kresse and D. Joubert, Phys. Rev. B: Condens. Matter Mater. Phys., 1999, 59, 1758-1775.

43 H. J. Monkhorst and J. D. Pack, Phys. Rev. B: Solid State, 1976, 13, 5188-5192.

44 C. E. Corbato, R. T. Tettenhorst and G. G. Christoph, Clays Clay Miner., 1985, 33, 71-75.

45 M. Digne, P. Sautet, P. Raybaud, P. Euzen and H. Toulhoat, J. Catal., 2002, 211, 1-5.

46 X. Krokidis, P. Raybaud, A. E. Gobichon, B. Rebours, P. Euzen and H. Toulhoat, J. Phys. Chem. B, 2001, 105, 5121-5130.

47 I. Levin and D. Brandon, J. Am. Ceram. Soc., 1998, 81, 19952012.

48 D. Tunega, H. Pasalic, M. H. Gerzabek and H. Lischka, J. Phys.: Condens. Matter, 2011, 23, 10.

49 K. S. Thanthiriwatte, E. G. Hohenstein, L. A. Burns and C. D. Sherrill, J. Chem. Theory Comput., 2011, 7, 88-96.

50 D. F. C. T. Office, Fuel Cell Technologies Office Multi-Year Research, Development, and Demonstration Plan, 2016, pp. 1-58.

51 T. U. S. D. Partnership, U.S. DRIVE Highlights of Technical Accomplishments, 2016, pp. 42-43. 